\title{
Envelope and Phase Distribution of Two Correlated Gaussian Variables
}

\author{
Prathapasinghe Dharmawansa, Member, IEEE, Nandana Rajatheva, Senior Member, IEEE, \\ and Chinthananda Tellambura, Senior Member, IEEE
}

\begin{abstract}
Probability density functions (pdf's) are derived for the phase and amplitude (envelope) of the complex gain $X+j Y$ $(j=\sqrt{-1})$, where $X$ and $Y$ are two correlated non zero-mean Gaussian random variables. The pdf of the amplitude is derived as an infinite series, but reduces to a closed-form expression when the means are zero. The classical Rayleigh and Rician pdf's turn out to be special cases of the derived pdf. This pdf is used to analyze the error performance of non-coherent binary frequency shift keying (BFSK) with in-phase/quadrature(I/Q) imbalance over an additive white Gaussian noise (AWGN) channel. The resulting bit error rate (BER) expression is derived as an infinite series. The analytical expressions are validated by simulation, and the $I / Q$ imbalance related performance degradation is quantified. Convergence of the PDF series and the BER series is established.
\end{abstract}

Index Terms-Characteristic function (chf), correlated Gaussian, frequency shift keying, in-phase/quadrature imbalance, probability density function (pdf), Rayleigh density.

\section{INTRODUCTION}

$\mathbf{W}$ HAT is the probability density function (pdf) of the amplitude (envelope) of the complex gain $X+j Y$ $(j=\sqrt{-1})$, where $X$ and $Y$ are two correlated non zero mean Gaussian random variables? Most wireless engineers are familiar with the answer to a special case of this question; i.e, when $X$ and $Y$ have zero correlation, one obtains the classical Rayleigh pdf or the Rician pdf, which are of fundamental importance not only in wireless communications, but also in many other fields of research. The pdf of the phase of the complex gain is of interest too.

Moreover, the pdf of $\sqrt{X^{2}+Y^{2}}$ is of interest as a generalization of the classical Rayleigh and Rician pdfs. It has application in in-phase/quadrature(I/Q) imbalance problems, which commonly occur in radio frequency receivers that employ analog quadrature down mixing, when there is a loss of orthogonality between the inphase and quadrature phase signals generated by the local oscillator. The effects of I/Q imbalance and the compensation methods are addressed in [1]-[3].

Paper approved by V. A. Aalo, the Editor for Diversity and Fading Channel Theory of the IEEE Communications Society. Manuscript received May 23, 2007; revised September 19, 2007 and December 7, 2007.

P. Dharmawansa was with the Telecommunications Field of Study, School of Engineering and Technology, Asian Institute of Technology, P. O. Box 4, Klong Luang, Pathumthani 12120, Thailand. He is now with the Department of Electronic and Computer Engineering, HKUST, Clear Water Bay, Kowloon, Hong Kong (e-mail: eesinghe@ust.hk).

N. Rajatheva is with the Telecommunications Field of Study, School of Engineering and Technology, Asian Institute of Technology, P. O. Box 4, Klong Luang, Pathumthani 12120, Thailand (e-mail: rajath@ait.ac.th).

C. Tellambura is with the Department of Electrical and Computer Engineering, University of Alberta, Edmonton, AB T6G 2V4, Canada (e-mail: chintha@ece.ualberta.ca).

Digital Object Identifier 10.1109/TCOMM.2009.04.070065
Beckmann [4] has addressed the derivation of this pdf. He notes that a pair of correlated variables can be transformed into a pair of independent ones by rotating through an angle. Based on this fact, he derives the pdf for the case of an independent but not identically distributed $X$ and $Y$. As a result, the pdf [4, 4.6-28] does not include the correlation coefficient explicitly. In [5] Pawla et al. introduced the distribution of phase angle between two vectors perturbed by correlated Gaussian noise. The Pawla $F$ function has been used to analyze the error performance of coherent M-ary phase shift keying (MPSK). Reference [6] derived a finite range integral expression for the symbol error rate (SER) of MPSK impaired with I/Q imbalance. The pdf expressions of the difference of phase angles in [5] were further modified to give new forms of expressions in [7]. A more compact set of equations involving Craig form of finite range integrals were derived in [8] for the SER performance of coherent MPSK modulation with I/Q imbalance. Vitthaladevuni and Alouini [9] introduced exact analytical bit error rate expressions of generalized hierarchical PSK constellations with imperfect phase or timing synchronization over an AWGN channel using Pawla $F$ functions. More general Nakagami-m distribution results are given in [10], [11].

The error performance of non-coherent detection of binary orthogonal, nonorthogonal signals is analyzed in [12], partially coherent detection in [13] and M-ary FSK in [14]. However, references [12]-[14] assume ideal I/Q balance. The conventional analysis of envelope detection of M-ary orthogonal signaling also assume an ideal I/Q balance. More realistic analysis of such systems requires that I/Q imbalance is fully accounted for. The pdf expressions derived in this paper may be useful in those problems.

Therefore, this letter derives the pdf's of the phase and amplitude (envelope) of the complex gain $X+j Y$, where $X$ and $Y$ are a pair of correlated Gaussian random variables. The pdf of the amplitude is derived as an infinite series, but reduces to a closed-form expression when the means are zero. The classical Rayleigh and Rician pdf's turn out to be special cases of the derived pdf. The results are applied to analyze the error performance of binary frequency shift keying (BFSK) with in-phase/quadrature(I/Q) imbalance over an additive white Gaussian noise (AWGN) channel. The resulting bit error rate (BER) expression is derived as an infinite series. The analytical expressions are validated by simulation, and the performance degradation due to the I/Q imbalance is quantified. Convergence of the PDF series and the BER series is established.

The paper is organized as follows. Section II derives the infinite series representations for the phase and amplitude 


$$
\begin{aligned}
p_{R}(r)= & \frac{r}{\sigma_{X} \sigma_{Y} \sqrt{1-\rho^{2}}} \exp \left\{-\frac{\left(\mu_{X}^{2} \sigma_{Y}^{2}+\mu_{Y}^{2} \sigma_{X}^{2}-2 \rho \mu_{X} \mu_{Y} \sigma_{X} \sigma_{Y}\right)}{2 \sigma_{X}^{2} \sigma_{Y}^{2}\left(1-\rho^{2}\right)}-\frac{\left(\sigma_{X}^{2}+\sigma_{Y}^{2}\right)}{4 \sigma_{X}^{2} \sigma_{Y}^{2}\left(1-\rho^{2}\right)} r^{2}\right\} \\
& \times \sum_{n=0}^{\infty} \varepsilon_{n} \cos \left(2 n\left(\beta-\frac{\psi}{2}\right)\right) I_{n}\left[\frac{\sqrt{\left(\sigma_{X}^{2}-\sigma_{Y}^{2}\right)^{2}+4 \rho^{2} \sigma_{X}^{2} \sigma_{Y}^{2}}}{4 \sigma_{X}^{2} \sigma_{Y}^{2}\left(1-\rho^{2}\right)} r^{2}\right] I_{2 n}\left[\frac{\sqrt{\Delta_{X, Y}}}{\sigma_{X}^{2} \sigma_{Y}^{2}\left(1-\rho^{2}\right)} r\right], \\
p_{\Theta}(\theta)= & \frac{\sigma_{X}^{3} \sigma_{Y}^{3} \sqrt{\left(1-\rho^{2}\right)^{3}}}{\pi \Delta_{\psi}(\theta)} \exp \left\{-\frac{\left(\mu_{X}^{2} \sigma_{Y}^{2}+\mu_{Y}^{2} \sigma_{X}^{2}-2 \rho \mu_{X} \mu_{Y} \sigma_{X} \sigma_{Y}\right)}{2 \sigma_{X}^{2} \sigma_{Y}^{2}\left(1-\rho^{2}\right)}\right\} \\
& \times\left\{1+\sqrt{\frac{\pi \Delta_{X, Y}}{\Delta_{\psi}(\theta)}} \cos (\theta-\beta) \operatorname{erfc}\left(-\sqrt{\frac{\Delta_{X, Y}}{\Delta_{\psi}(\theta)}} \cos (\theta-\beta)\right) \exp \left(\frac{\Delta_{X, Y}}{\Delta_{\psi}(\theta)} \cos ^{2}(\theta-\beta)\right)\right\}
\end{aligned}
$$

$$
\begin{aligned}
f_{\Theta}(\theta) & =\frac{\sqrt{1-\rho^{2}}}{2 \pi(1-\rho \sin 2 \theta)} \exp \left\{-\frac{\left(\mu_{X}^{2}+\mu_{Y}^{2}-2 \mu_{X} \mu_{Y} \rho\right)}{2 \sigma^{2}\left(1-\rho^{2}\right)}\right\}\left\{\sqrt { \frac { \pi \Omega _ { X , Y } } { \Omega ( \theta ) } } \operatorname { c o s } ( \theta - \phi ) \operatorname { e r f c } \left(-\sqrt{\left.\frac{\Omega_{X, Y}}{\Omega(\theta)} \cos (\theta-\phi)\right)}\right.\right. \\
& \left.\times \exp \left(\frac{\Omega_{X, Y}}{\Omega(\theta)} \cos ^{2}(\theta-\phi)\right)+1\right\}
\end{aligned}
$$

(envelope) of the complex gain $X+j Y$, where $X$ and $Y$ are two correlated non zero-mean Gaussian random variables. Special cases of the general results are identified. In Section III, the BER performance of non-coherent BFSK in AWGN is analyzed. Section IV summarizes the main results of the paper. The derivation of the infinite series and convergence results are presented in the appendices.

\section{PDF'S OF THE AMPLITUDE AND PHASE OF A PAIR OF CORRELATED GAUSSIAN RANDOM VARIABLES}

The key results on the distributions of the envelope and phase are now presented.

Theorem 1: Let $X$ and $Y$ be two real Gaussian random variables with $\operatorname{VAR}[X]=\sigma_{X}^{2}, \operatorname{VAR}[Y]=\sigma_{Y}^{2}$ and mean values $E[X]=\mu_{X}$ and $E[Y]=\mu_{Y}$. The correlation coefficient between $X$ and $Y$ is $\rho$. The pdf of $R=\sqrt{X^{2}+Y^{2}}$ is given by (1) and the distribution of $\Theta=\tan ^{-1}\left(\frac{Y}{X}\right)$ is given by (2), where $\Delta_{X, Y}=\mu_{X}^{2} \sigma_{Y}^{4}+\mu_{Y}^{2} \sigma_{X}^{4}+\rho^{2} \sigma_{X}^{2} \sigma_{Y}^{2}\left(\mu_{X}^{2}+\mu_{Y}^{2}\right)-$ $2 \rho \mu_{X} \mu_{Y} \sigma_{X} \sigma_{Y}\left(\sigma_{X}^{2}+\sigma_{Y}^{2}\right)$

$$
\begin{aligned}
\cos \psi & =\frac{\sigma_{X}^{2}-\sigma_{Y}^{2}}{\sqrt{\left(\sigma_{X}^{2}-\sigma_{Y}^{2}\right)^{2}+4 \rho^{2} \sigma_{X}^{2} \sigma_{Y}^{2}}} \\
\sin \psi & =\frac{2 \rho \sigma_{x} \sigma_{Y}}{\sqrt{\left(\sigma_{X}^{2}-\sigma_{Y}^{2}\right)^{2}+4 \rho^{2} \sigma_{X}^{2} \sigma_{Y}^{2}}} \\
\cos \beta & =\frac{\sigma_{Y}\left(\mu_{X} \sigma_{Y}-\rho \mu_{Y} \sigma_{X}\right)}{\sqrt{\Delta_{X, Y}}} \\
\sin \beta & =\frac{\sigma_{X}\left(\mu_{Y} \sigma_{X}-\rho \mu_{X} \sigma_{Y}\right)}{\sqrt{\Delta_{X, Y}}} \\
\Delta_{\psi}(\theta)=\sigma_{X}^{2} \sigma_{Y}^{2}\left(1-\rho^{2}\right) & {\left[\sigma_{X}^{2}+\sigma_{Y}^{2}-\sqrt{\left(\sigma_{X}^{2}-\sigma_{Y}^{2}\right)^{2}+4 \rho^{2} \sigma_{X}^{2} \sigma_{Y}^{2}} \cos (2 \theta-\psi)\right], }
\end{aligned}
$$

$\operatorname{erfc}(z)=\frac{2}{\sqrt{\pi}} \int_{z}^{\infty} \exp \left(-t^{2}\right) d t$ is the complementary error function, $\varepsilon_{n}$ is the Neumann factor [15] (i.e., $\varepsilon_{0}=1$ and $\varepsilon_{n}=2$ for all $\left.n=1,2, \ldots.\right)$ and $I_{n}(z)$ is the $n$-th order modified Bessel function of the first kind.

Proof: See Appendix A.

The above envelope density function can easily be reduced to [4, eq.4.6-28] when $\rho=0$. Furthermore, the distribution of phase generalizes most of the results given in the literature including [16, eq. D.105] (which can easily be derived assuming $\rho=0$ and $\left.\mu_{Y}=0\right)$.

Corollary 1: Let $X$ and $Y$ be two real Gaussian random variables with $\operatorname{VAR}[X]=\operatorname{VAR}[Y]=\sigma^{2}$ and mean values $E[X]=\mu_{X}$ and $E[Y]=\mu_{Y}$. The correlation coefficient between $X$ and $Y$ is $\rho$. The pdf of $R=\sqrt{X^{2}+Y^{2}}$ is given by

$$
\begin{aligned}
f_{R}(r)= & \frac{r}{\sigma^{2} \sqrt{1-\rho^{2}}} \exp \left\{-\frac{\left(\mu_{X}^{2}+\mu_{Y}^{2}-2 \mu_{X} \mu_{Y} \rho\right)+r^{2}}{2 \sigma^{2}\left(1-\rho^{2}\right)}\right\} \\
& \times \sum_{n=0}^{\infty} \varepsilon_{n} \cos \left(2 n\left(\phi-\frac{\pi}{4}\right)\right) I_{n}\left[\frac{\rho r^{2}}{2 \sigma^{2}\left(1-\rho^{2}\right)}\right] \\
& \times I_{2 n}\left[\frac{\sqrt{\Omega_{X, Y}}}{\sigma^{2}\left(1-\rho^{2}\right)} r\right.
\end{aligned}
$$

and the distribution of $\Theta=\tan ^{-1}\left(\frac{Y}{X}\right)$ is given by (4) above, where $\Omega_{X, Y}=\left(\mu_{X}^{2}+\mu_{Y}^{2}\right)\left(1+\rho^{2}\right)-4 \mu_{X} \mu_{Y} \rho, \Omega(\theta)=$ $2 \sigma^{2}\left(1-\rho^{2}\right)(1-\rho \sin 2 \theta), \cos \phi=\frac{\mu_{X}-\mu_{Y} \rho}{\sqrt{\Omega_{X, Y}}}$ and $\sin \phi=$ $\frac{\mu_{Y}-\mu_{X} \rho}{\sqrt{\Omega_{X, Y}}}$.

Proof: Substituting $\sigma_{X}=\sigma_{Y}=\sigma$ in (1) and (2) gives $\psi=\pi / 2$, and (3) and (4) follow.

Corollary 2: Let $X$ and $Y$ be two real identical Gaussian random variables with $\operatorname{VAR}[X]=\operatorname{VAR}[Y]=\sigma^{2}$ and mean values $E[X]=E[Y]=\mu$. The correlation coefficient between 


$$
\begin{aligned}
P_{R}(r) & =\frac{2 \sigma_{X} \sigma_{Y} \sqrt{1-\rho^{2}}}{\sigma_{X}^{2}+\sigma_{Y}^{2}} \exp \left\{-\frac{\left(\mu_{X}^{2} \sigma_{Y}^{2}+\mu_{Y}^{2} \sigma_{X}^{2}-2 \rho \mu_{X} \mu_{Y} \sigma_{X} \sigma_{Y}\right)}{2 \sigma_{X}^{2} \sigma_{Y}^{2}\left(1-\rho^{2}\right)}\right\} \sum_{n, p, q=0}^{\infty} \frac{\varepsilon_{n} \cos 2 n\left(\beta-\frac{\psi}{2}\right)}{p ! q !(n+p) !(2 n+q) !} \\
& \times\left(\frac{\left(\sigma_{X}^{2}-\sigma_{Y}^{2}\right)^{2}+4 \rho^{2} \sigma_{X}^{2} \sigma_{Y}^{2}}{4\left(\sigma_{X}^{2}+\sigma_{Y}^{2}\right)^{2}}\right)^{p+\frac{n}{2}}\left(\frac{\Omega_{X, Y}}{\sigma_{X}^{2} \sigma_{Y}^{2}\left(1-\rho^{2}\right)\left(\sigma_{X}^{2}+\sigma_{Y}^{2}\right)}\right)^{q+n} \gamma\left(2 p+2 n+q+1, \frac{\sigma_{X}^{2}+\sigma_{Y}^{2}}{4 \sigma_{X}^{2} \sigma_{Y}^{2}\left(1-\rho^{2}\right)} r^{2}\right)
\end{aligned}
$$

$X$ and $Y$ is $\rho$. The pdf of $R=\sqrt{X^{2}+Y^{2}}$ is given by

$$
\begin{aligned}
g_{R}(r) & =\frac{r}{\sigma^{2} \sqrt{1-\rho^{2}}} \exp \left\{-\frac{2 \mu^{2}(1-\rho)+r^{2}}{2 \sigma^{2}\left(1-\rho^{2}\right)}\right\} \\
& \times \sum_{n=0}^{\infty} \varepsilon_{n} I_{n}\left[\frac{\rho r^{2}}{2 \sigma^{2}\left(1-\rho^{2}\right)}\right] I_{2 n}\left[\frac{\sqrt{2 \mu^{2}}}{\sigma^{2}(1+\rho)} r\right] .
\end{aligned}
$$

Proof: According to (3) $\phi=\frac{\pi}{4}$ when $\mu_{X}=\mu_{Y}=\mu$. Hence by substituting them in (3) gives (5).

Corollary 3: Let $X$ and $Y$ be two real identical Gaussian random variables with $\operatorname{VAR}[X]=\operatorname{VAR}[Y]=\sigma^{2}$ and mean values $E[X]=E[Y]=0$. The correlation coefficient between $X$ and $Y$ is $\rho$. The pdf of $R=\sqrt{X^{2}+Y^{2}}$ is given by

$$
h_{R}(r)=\frac{r \exp \left\{-\frac{r^{2}}{2 \sigma^{2}\left(1-\rho^{2}\right)}\right\}}{\sigma^{2} \sqrt{1-\rho^{2}}} I_{0}\left[\frac{\rho r^{2}}{2 \sigma^{2}\left(1-\rho^{2}\right)}\right] \text {. }
$$

Proof: By substituting zero for $\mu$ in (5) and using $I_{0}(0)=$ 1 , the result follows.

The cumulative distribution function (cdf) of $R$ for the case described in Theorem 1 can easily be derived with the series expansion of each of the Bessel function terms in (1) followed by term by term integration. Thus, the cdf of $R$ is obtained as given at the top of the page, where $\gamma(a, z)=$ $\int_{0}^{z} t^{a-1} \exp (-t) d t$ is the incomplete gamma function.

Note that the classical results for Rayleigh (i.e., when $\mu_{X}=$ $\mu_{Y}=0, \sigma_{X}=\sigma_{Y}=\sigma$ and $\rho=0$ ) and Rician densities (i.e., when $\rho=0$ and $\sigma_{X}=\sigma_{Y}=\sigma$ with the Rician factor $K=\frac{\mu_{X}^{2}+\mu_{Y}^{2}}{2 \sigma^{2}}$ ) are some special cases of (1).

Figure 1 depicts the theoretical pdf, $g_{R}(r)$ calculated with (5) and the simulated values for different values of $\alpha$ (here, without loss of generality, $\rho=\sin \alpha$ ) with $\mu=1, \sigma^{2}=1$.

\section{The BER Performance of Non-COHEREnt BFSK WITH I/Q IMBALANCE}

This section presents an illustrative application of the preceding results. Although only one application is considered for brevity, potential others include performance analysis of $M$-ary FSK under I/Q imbalance.

Consider a noncoherent BFSK system which employs two carrier frequencies $f_{1}, f_{2}$ with $f_{2}-f_{1}=\frac{1}{T}$, where $T$ is the bit duration. This minimum frequency difference criterion ensures that the carriers are orthogonal [17]. Let us consider the simplified block diagram of the BFSK receiver shown in Fig. 2, where $\alpha$ represents the I/Q imbalance phase. For simplicity, the I/Q imbalance phase angles are assumed to be identical for both the carrier frequencies. The received signal can be written as

$$
r(t)=\sqrt{\frac{2 E_{b}}{T}} \cos \left(2 \pi f_{i} t+\varphi\right)+n(t), \quad i=1,2
$$

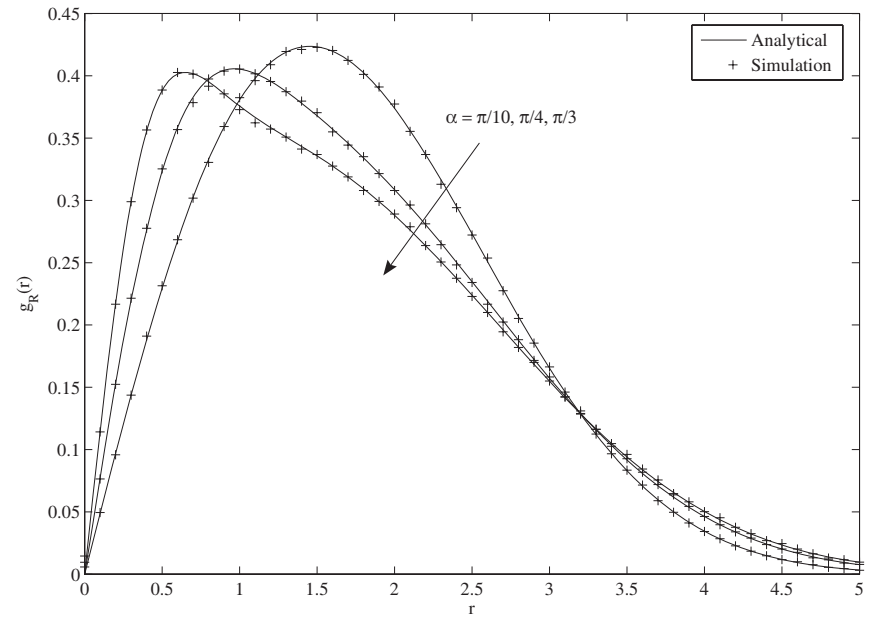

Fig. 1. The pdf of $g_{R}(r)$ for different values of $\rho=\sin \alpha$ when $\mu=1$ and $\sigma^{2}=1$.

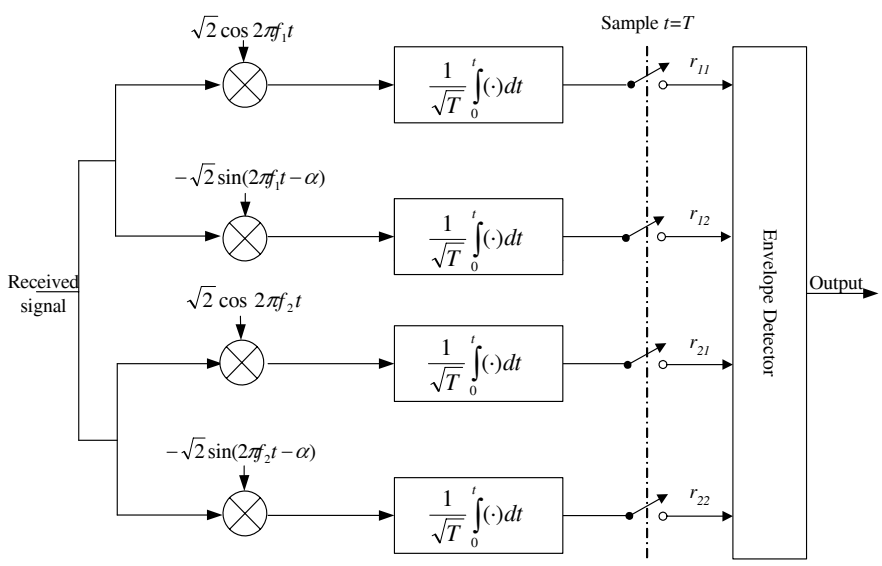

Fig. 2. Block diagram of the non-coherent BFSK receiver.

where $\varphi$ is a random phase angle uniformly distributed over $[0,2 \pi), E_{b}$ denotes the energy per BFSK symbol and $n(t)$ denotes a white Gaussian noise process having the autocorrelation function $R_{n}(\tau)=\frac{N_{0}}{2} \delta(\tau)$. Without loss of generality, we assume that $f_{1}$ and $f_{2}$ are equiprobable and that $f_{1}$ is transmitted. The sample values at the input to the envelope detector as $r_{11}=\sqrt{E_{b}} \cos \varphi+n_{11}, r_{12}=\sqrt{E_{b}} \sin (\varphi+\alpha)+n_{12}$ and $r_{21}=n_{21}, r_{22}=n_{22}$ where $\left(n_{11}, n_{12}\right)$ and $\left(n_{21}, n_{22}\right)$ are independent Gaussian random pairs while the random variables in each pair are jointly Gaussian with variance $\frac{N_{0}}{2}$ and correlation coefficient $\rho=\sin \alpha$. An error occurs if $\sqrt{r_{11}^{2}+r_{12}^{2}}<\sqrt{r_{21}^{2}+r_{22}^{2}}$ or the normalized variables satisfy $\frac{\sqrt{r_{11}^{2}+r_{12}^{2}}}{\sqrt{N_{0} / 2}}\left(=R_{1}\right)<\frac{\sqrt{r_{21}^{2}+r_{22}^{2}}}{\sqrt{N_{0} / 2}}\left(=R_{2}\right)$ [17]. Following [17], 
the conditional BER of BFSK can be expressed as

$$
P(\epsilon \mid \varphi)=\int_{0}^{\infty} \int_{r_{1}}^{\infty} f_{R_{2}}\left(r_{2}\right) d r_{2} f_{R_{1}}\left(r_{1}\right) d r_{1}
$$

where $f_{R_{1}}\left(r_{1}\right)$ can be written using (3) as (here $\mu_{X}=$ $\sqrt{E_{b}} \cos \varphi, \mu_{Y}=\sqrt{E_{b}} \sin (\varphi+\alpha)$ and $\left.\sigma^{2}=\frac{N_{0}}{2}\right)$

$$
\begin{aligned}
& f_{R_{1}}\left(r_{1}\right)=r_{1}|\sec \alpha| \exp \left(-\gamma_{b}-\frac{r_{1}^{2} \sec ^{2} \alpha}{2}\right) \\
& \quad \times \sum_{n=0}^{\infty} \varepsilon_{n} \cos 2 n\left(\beta-\frac{\pi}{4}\right) I_{n}\left(\frac{\sin \alpha \sec ^{2} \alpha}{2} r_{1}^{2}\right) \\
& \quad \times I_{2 n}\left(\sec \alpha \sqrt{2 \gamma_{b}\left(\sin ^{2} \varphi+\cos ^{2}(\alpha+\varphi)\right)} r_{1}\right),
\end{aligned}
$$

$f_{R_{2}}\left(r_{2}\right)$ can be expressed using (6) as

$$
\begin{gathered}
f_{R_{2}}\left(r_{2}\right)=r_{2}|\sec \alpha| \exp \left(-\frac{r_{2}^{2} \sec ^{2} \alpha}{2}\right) I_{0}\left(\frac{\sin \alpha \sec ^{2} \alpha}{2} r_{2}^{2}\right) \\
\cos \beta=\frac{\cos (\alpha+\varphi)}{\sqrt{\sin ^{2} \varphi+\cos ^{2}(\alpha+\varphi)}} \\
\sin \beta=\frac{\sin \varphi}{\sqrt{\sin ^{2} \varphi+\cos ^{2}(\alpha+\varphi)}}
\end{gathered}
$$

where $\gamma_{b}=\frac{E_{b}}{N_{0}}$. Since a closed-form solution does not exist for the inner integral in (8), an infinite series solution is sought. Using the equivalent infinite series representation of modified Bessel functions and [18, eq.3.351.2], the inner integral can be written as

$$
\begin{aligned}
\int_{r_{1}}^{\infty} f_{R_{2}}\left(r_{2}\right) d r_{2}=\sum_{k=0}^{\infty} \sum_{l=0}^{2 k} \frac{(2 k) !\left|\sec ^{2 l-1} \alpha\right| \sin ^{2 k} \alpha}{l !(k !)^{2} 2^{2 k+l}} r_{1}^{2 l} \\
\\
\quad \times \exp \left(-\frac{r_{1}^{2} \sec ^{2} \alpha}{2}\right) .
\end{aligned}
$$

By substituting (11) and (9) in (8) followed by the expansion of Bessel function terms with equivalent infinite series and term-by-term integration, the conditional BER is obtained as

$$
\begin{aligned}
& P(\epsilon \mid \varphi)=\sum_{k=0}^{\infty} \sum_{l=0}^{2 k} \sum_{n, p, q=0}^{\infty} \frac{\varepsilon_{n}(2 k) ! \eta_{2} ! \sin ^{\eta_{1}} \alpha \cos ^{2} \alpha \gamma^{q+n}}{(k !)^{2} l ! p ! q !(p+n) !(q+2 n) !} \\
& \times \frac{\exp (-\gamma) \cos 2 n\left(\beta-\frac{\pi}{4}\right)}{2^{\eta_{1}+\eta_{2}+1}}\left[\sin ^{2} \varphi+\cos ^{2}(\alpha+\varphi)\right]^{q+n}
\end{aligned}
$$

where $\eta_{1}=2 k+2 p+n, \eta_{2}=2 p+2 n+l+q$. The form of conditional BER given in (12) is important in the sense of obtaining the error rate expression in the absence of I/Q imbalance. When the inphase and quadrature components are phase shifted by $\pi / 2$ (i.e., $\alpha=0$ ) the nested summations in (12) degenerate into the following simple form

$$
P(\epsilon \mid \varphi)=\frac{\exp (-\gamma)}{2} \sum_{q=0}^{\infty} \frac{\gamma^{q}}{2^{q} q !}
$$

which can easily be integrated with respect to $\varphi$ to obtain the classical expression for BER of the noncoherent BFSK as $\frac{1}{2} \exp \left(-\frac{\gamma}{2}\right)$ [17]. With the uniformly distributed random phase, (12) yields the BER as

$$
\begin{aligned}
P(\epsilon \mid \varphi)= & \sum_{k=0}^{\infty} \sum_{l=0}^{2 k} \sum_{n, p, q=0}^{\infty} \frac{\varepsilon_{n}(2 k) ! \eta_{2} ! \sin ^{\eta_{1}} \alpha \cos ^{2} \alpha \gamma^{q+n}}{(k !)^{2} l ! p ! q !(p+n) !(q+2 n) !} \\
& \times \frac{\exp (-\gamma)}{2^{\eta_{1}+\eta_{2}+2} \pi} \int_{0}^{2 \pi} \cos 2 n\left(\beta-\frac{\pi}{4}\right) \\
& \times\left[\sin ^{2} \varphi+\cos ^{2}(\alpha+\varphi)\right]^{q+n} d \varphi .
\end{aligned}
$$

Now let us consider the integral in (14) which can be expressed using (10) as

$$
\begin{aligned}
I(\alpha)= & \int_{0}^{2 \pi} \cos 2 n\left(\beta-\frac{\pi}{4}\right)\left[\sin ^{2} \varphi+\cos ^{2}(\alpha+\varphi)\right]^{q+n} d \varphi \\
= & \cos \frac{n \pi}{2} \sum_{s=0}^{n}(-1)^{s}\left(\begin{array}{c}
2 n \\
2 s
\end{array}\right) J(2 s, 2 n-2 s) \\
& +\sin \frac{n \pi}{2} \sum_{s=0}^{n-1}(-1)^{s}\left(\begin{array}{c}
2 n \\
2 s+1
\end{array}\right) J(2 s+1,2 n-2 s-1)
\end{aligned}
$$

where

$$
\begin{aligned}
J(\alpha, u, v)=\int_{0}^{2 \pi} \sin ^{u} \varphi \cos ^{v}(\alpha+\varphi) & \\
& {\left[\sin ^{2} \varphi+\cos ^{2}(\alpha+\varphi)\right]^{q} d \varphi }
\end{aligned}
$$

with $u, v=0,1,2, .$. and $\left(\begin{array}{c}m \\ n\end{array}\right)$ denotes the binomial coefficient. Here, the $\cos 2 n \beta$ and $\sin 2 n \beta$ terms have been expanded using cosine and sine terms. The integral given in (16) can further be decomposed using the binomial theorem into simple integrals involving sine and cosine terms. However, this would increase the number of summations and hence affect the computational complexity. Taking this difficulty into account, (15) and (16) in (14) and numerical integration techniques can be used to find the exact BER values. This allows a tradeoff between analytical tractability and numerical efficiency.

Next, our theoretical results are validated by using the simulation results (Fig. 3). A noncoherent BFSK in AWGN is simulated, and the numerical results based on (14) are compared with the simulation results. A span of $10 \mathrm{~dB}$ is selected along SNR axis and $\alpha=0, \pi / 10, \pi / 8$ for calculating the theoretical results with (14). The degree of accuracy depends on the number of terms used to truncate the infinite series. Since the increase in either of the quantities $\gamma$ or $\alpha$ would increase the terms to be summed with (14), we have selected SNR and $\alpha$ values mentioned above. We use a minimum of $7,13,7,7$ and a maximum of $23,13,23,23$ terms in each index of $k, n, p, q$ respectively. Mathematica is used to perform the numerical integrations given in (15). Fig. 3 shows that our analytical results agree well with the simulation results. The performance degradation due to the I/Q imbalance increases with the increase of mismatch, as to be expected.

\section{CONCLUSION}

This letter has derived pdf expressions for the phase and amplitude (envelope) of the complex gain $X+j Y$, where $X$ and $Y$ are two correlated non zero mean Gaussian random variables. The classical Rayleigh and Rician pdf's are special 


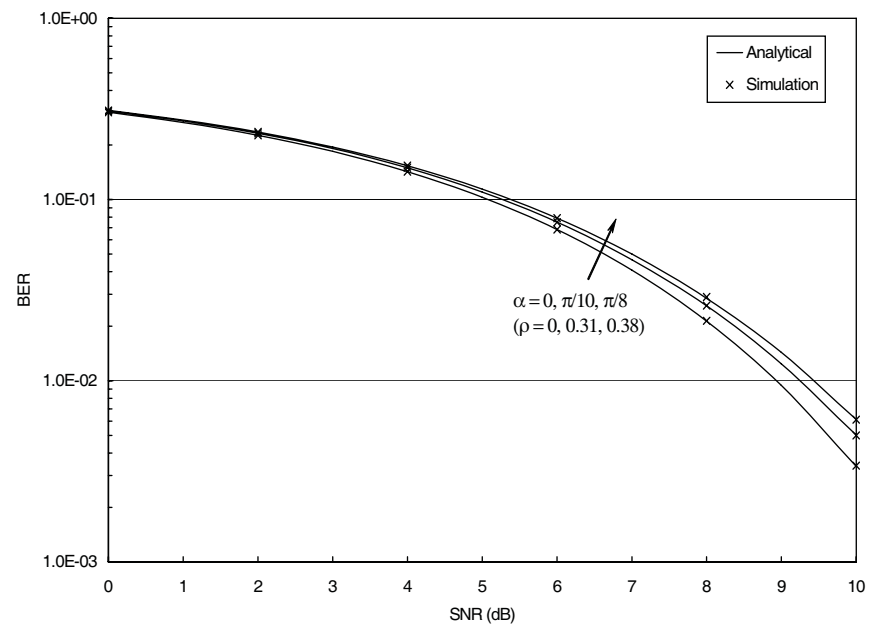

Fig. 3. BER performance of the non-coherent BFSK receiver with I/Q imbalance.

cases of the derived pdf. Convergence results have been established. The pdf has been used to analyze the error performance of BFSK with I/Q imbalance over an AWGN channel. This analysis can also be extended to $M$-ary FSK $(M>2)$. The analytical expressions were validated by simulation. The pdf expressions may also be useful for other I/Q imbalance problems and/or correlated Gaussian noise problems.

\section{APPENDIX A}

\section{PROOF OF THEOREM 1}

Using the classical rectangular-to-polar coordinate transformation (i.e., $x=r \cos \theta, y=r \sin \theta$ ) the joint pdf of two correlated Gaussian random variables $X, Y$ having parameters $\mu_{X}, \mu_{Y}, \sigma_{X}^{2}, \sigma_{Y}^{2}, \rho$ with $|\rho|<1$ can be written as

$$
\begin{aligned}
& p_{R, \Theta}(r, \theta)=\frac{r e^{\left\{-\frac{\left(\mu_{X}^{2} \sigma_{Y}^{2}+\mu_{Y}^{2} \sigma_{X}^{2}-2 \rho \mu_{X} \mu_{Y} \sigma_{X} \sigma_{Y}\right)}{2 \sigma_{X}^{2} \sigma_{Y}^{2}\left(1-\rho^{2}\right)}\right\}}}{2 \pi \sigma_{X} \sigma_{Y} \sqrt{1-\rho^{2}}} \\
& \left.\times e^{\left\{-\frac{\left(\sigma_{X}^{2}+\sigma_{Y}^{2}\right)}{4 \sigma_{X}^{2} \sigma_{Y}^{2}\left(1-\rho^{2}\right)} r^{2}\right.}\right\} e^{\left[\sqrt{A^{2}(r)+B^{2}(r)} \cos (2 \theta-\psi)\right]} \\
& \times e^{\left[\sqrt{C^{2}(r)+D^{2}(r)} \cos (\theta-\beta)\right]}
\end{aligned}
$$

where $A(r)=\frac{\rho r^{2}}{2 \sigma_{X} \sigma_{Y}\left(1-\rho^{2}\right)}, B(r)=\frac{\left(\sigma_{X}^{2}-\sigma_{Y}^{2}\right) r^{2}}{4 \sigma_{X}^{2} \sigma_{Y}^{2}\left(1-\rho^{2}\right)}, C(r)=$ $\frac{\left(\mu_{X} \sigma_{Y}-\rho \mu_{Y} \sigma_{X}\right) r}{\sigma_{X}^{2} \sigma_{Y}\left(1-\rho^{2}\right)}, D(r)=\frac{\left(\mu_{Y} \sigma_{X}-\rho \mu_{X} \sigma_{Y}\right) r}{\sigma_{Y}^{2} \sigma_{X}\left(1-\rho^{2}\right)}$ and $\psi, \beta$ are defined in Section II. To find the marginal pdf of $R$, (17) must be integrated with respect to $\theta \in[0,2 \pi)$. By observing the periodicity of the composite trigonometric expression within the exponential term in (17) and using the following identity [19]

$$
\exp (z \cos \theta)=\sum_{n=0}^{\infty} \varepsilon_{n} I_{n}(z) \cos n \theta
$$

$R$ can be written as

$$
\begin{aligned}
p_{R}(r) & =\frac{r}{2 \pi \sigma_{X} \sigma_{Y} \sqrt{1-\rho^{2}}} e^{\left\{-\frac{\left(\mu_{X}^{2} \sigma_{Y}^{2}+\mu_{Y}^{2} \sigma_{X}^{2}-2 \rho \mu_{X} \mu_{Y} \sigma_{X} \sigma_{Y}\right)}{2 \sigma_{X}^{2} \sigma_{Y}^{2}\left(1-\rho^{2}\right)}\right\}} \\
& \left.\times e^{\left\{-\frac{\left(\sigma_{X}^{2}+\sigma_{Y}^{2}\right)}{4 \sigma_{X}^{2} \sigma_{Y}^{2}\left(1-\rho^{2}\right)} r^{2}\right.}\right\} \sum_{n=0}^{\infty} \varepsilon_{n} I_{n}\left[\sqrt{A^{2}(r)+B^{2}(r)}\right] \\
& \times \int_{0}^{2 \pi} \cos 2 n\left(\theta+\beta-\frac{\psi}{2}\right) e^{\left[\sqrt{C^{2}(r)+D^{2}(r)} \cos \theta\right]} d \theta .
\end{aligned}
$$

Next, the $\cos 2 n\left(\theta+\beta-\frac{\psi}{2}\right)$ term is expanded as a sum of product of cosine and sine terms and observing the fact that the sine is an odd function followed by the integration using [19, eq.7.34] gives the desired result(1).

Next we prove (2) starting with (17). By rearranging the terms in (17), the joint pdf becomes

$$
\begin{aligned}
& p_{R, \Theta}(r, \theta)=\frac{e^{\left\{-\frac{\left(\mu_{X}^{2} \sigma_{Y}^{2}+\mu_{Y}^{2} \sigma_{X}^{2}-2 \rho \mu_{X} \mu_{Y} \sigma_{X} \sigma_{Y}\right)}{2 \sigma_{X}^{2} \sigma_{Y}^{2}\left(1-\rho^{2}\right)}\right\}}}{2 \pi \sigma_{X} \sigma_{Y} \sqrt{1-\rho^{2}}} \\
& \times r e^{\left(-R(\theta) r^{2}+S(\theta) r\right)}
\end{aligned}
$$

where $R(\theta)=\frac{\Delta_{\psi}(\theta)}{4 \sigma_{X}^{2} \sigma_{Y}^{2}\left(1-\rho^{2}\right)}$ and $S(\theta)=\frac{\sqrt{\Delta_{X, Y}}}{\sigma_{X}^{2} \sigma_{Y}^{2}\left(1-\rho^{2}\right)} \cos (\theta-$ $\beta$ ). Now it is obvious that (19) has to be integrated with respect to $r$ to obtain the marginal of $\Theta$. Completing the square of the quadratic expression in the exponent and subsequent integration with respect to $r$ yields the marginal of $\Theta$ as (2).

\section{APPENDIX B \\ Convergence of INFINITE SERIES}

\section{A. Convergence of (1)}

Before proving the convergence, we establish the following inequality.

$$
I_{n}(z)<I_{0}(z), \quad n=1,2, \ldots .
$$

From the definition of $I_{n}(z)$, it follows that

$$
\begin{aligned}
I_{n}(z)= & \frac{1}{\pi} \int_{0}^{\pi} \exp (z \cos \theta) \cos n \theta d \theta \\
& <\frac{1}{\pi} \int_{0}^{\pi} \exp (z \cos \theta)|\cos n \theta| d \theta .
\end{aligned}
$$

Since $|\cos n \theta|<1$, the inequality (20) follows immediately. Next we show that the infinite series (1) converges absolutely. To this end, (1) is transformed as

$$
\begin{aligned}
&\left|p_{R}(r)\right|<\frac{r e^{-\frac{\left(\mu_{X}^{2} \sigma_{Y}^{2}+\mu_{Y}^{2} \sigma_{X}^{2}-2 \rho \mu_{X} \mu_{Y} \sigma_{X} \sigma_{Y}\right)}{2 \sigma_{X}^{2} \sigma_{Y}^{2}\left(1-\rho^{2}\right)}-\frac{\left(\sigma_{X}^{2}+\sigma_{Y}^{2}\right)}{4 \sigma_{X}^{2} \sigma_{Y}^{2}\left(1-\rho^{2}\right)} r^{2}}}{\sigma_{X} \sigma_{Y} \sqrt{1-\rho^{2}}} \\
& \times \sum_{n=0}^{\infty} \varepsilon_{n} I_{n}\left[\frac{\sqrt{\left(\sigma_{X}^{2}-\sigma_{Y}^{2}\right)^{2}+4 \rho^{2} \sigma_{X}^{2} \sigma_{Y}^{2}}}{4 \sigma_{X}^{2} \sigma_{Y}^{2}\left(1-\rho^{2}\right)} r^{2}\right], \\
& \times I_{2 n}\left[\frac{\sqrt{\Delta_{X, Y}}}{\sigma_{X}^{2} \sigma_{Y}^{2}\left(1-\rho^{2}\right)} r\right]
\end{aligned}
$$


and applying (20) it becomes

$$
\begin{aligned}
\left|p_{R}(r)\right|< & \frac{r e^{-\frac{\left(\mu_{X}^{2} \sigma_{Y}^{2}+\mu_{Y}^{2} \sigma_{X}^{2}-2 \rho \mu_{X} \mu_{Y} \sigma_{X} \sigma_{Y}\right)}{2 \sigma_{X}^{2} \sigma_{Y}^{2}\left(1-\rho^{2}\right)}-\frac{\left(\sigma_{X}^{2}+\sigma_{Y}^{2}\right)}{4 \sigma_{X}^{2} \sigma_{Y}^{2}\left(1-\rho^{2}\right)} r^{2}}}{\sigma_{X} \sigma_{Y} \sqrt{1-\rho^{2}}} \\
& \times I_{0}\left[\frac{\sqrt{\Delta_{X, Y}}}{\sigma_{X}^{2} \sigma_{Y}^{2}\left(1-\rho^{2}\right)} r\right] \\
& \times \sum_{n=0}^{\infty} \varepsilon_{n} I_{n}\left[\frac{\sqrt{\left(\sigma_{X}^{2}-\sigma_{Y}^{2}\right)^{2}+4 \rho^{2} \sigma_{X}^{2} \sigma_{Y}^{2}}}{4 \sigma_{X}^{2} \sigma_{Y}^{2}\left(1-\rho^{2}\right)} r^{2}\right] .
\end{aligned}
$$

Now using the relation (20) it is easy to see that the infinite summation converges to $\exp \left\{\frac{\sqrt{\left(\sigma_{X}^{2}-\sigma_{Y}^{2}\right)^{2}+4 \rho^{2} \sigma_{X}^{2} \sigma_{Y}^{2}}}{4 \sigma_{X}^{2} \sigma_{Y}^{2}\left(1-\rho^{2}\right)} r^{2}\right\}$ and hence we have

$$
\begin{aligned}
\left|p_{R}(r)\right|< & \frac{r e^{-\frac{\left(\mu_{X}^{2} \sigma_{Y}^{2}+\mu_{Y}^{2} \sigma_{X}^{2}-2 \rho \mu_{X} \mu_{Y} \sigma_{X} \sigma_{Y}\right)}{2 \sigma_{X}^{2} \sigma_{Y}^{2}\left(1-\rho^{2}\right)}-\frac{\left(\sigma_{X}^{2}+\sigma_{Y}^{2}\right)}{4 \sigma_{X}^{2} \sigma_{Y}^{2}\left(1-\rho^{2}\right)} r^{2}}}{\sigma_{X} \sigma_{Y} \sqrt{1-\rho^{2}}} \\
& \times e^{-\frac{\Delta_{0}(0)}{4 \sigma_{X}^{4} \sigma_{Y}^{4}\left(1-\rho^{2}\right)^{2}} r^{2}} I_{0}\left[\frac{\sqrt{\Delta_{X, Y}}}{\sigma_{X}^{2} \sigma_{Y}^{2}\left(1-\rho^{2}\right)} r\right]
\end{aligned}
$$

which completes our proof. Since we proved that the absolute sum is upper bounded with a continuous function, the uniform convergence of infinite series (1) can be assumed without loss of generality.

\section{B. Convergence of (14)}

The following result is worth of mentioning before a detailed discussion on the convergence of (14).

For $L=0,1,2 \ldots$ we can write

$$
\sum_{n=0}^{\infty} \frac{(n+L) !}{n ! 2^{n}}=L !_{1} F_{0}\left(L+1 ;-; \frac{1}{2}\right)=L ! 2^{L+1}
$$

where ${ }_{p} F_{q}\left(a_{1}, a_{2}, \cdots, a_{p} ; c_{1}, c_{2}, \cdots, c_{q} ; x\right)$ is the generalized hypergeometric function [19]. In what follows we show that the series (14) converges absolutely. It is easy to see that (14) can easily be written as

$$
\begin{aligned}
|P(\epsilon \mid \varphi)|< & \sum_{k=0}^{\infty} \sum_{l=0}^{2 k} \sum_{n, p, q=0}^{\infty} \frac{\varepsilon_{n}(2 k) ! \eta_{2} !\left|\sin ^{\eta_{1}} \alpha\right| \cos ^{2} \alpha}{(k !)^{2} l ! p ! q !(p+n) !(q+2 n) !} \\
& \frac{\gamma^{q+n} \exp (-\gamma)}{2^{\eta_{1}+\eta_{2}+1}}\left[\sin ^{2} \varphi+\cos ^{2}(\alpha+\varphi)\right]^{q+n} .
\end{aligned}
$$

The right side of above inequality can be upper bounded as

$$
\begin{aligned}
&|P(\epsilon \mid \varphi)|< \sum_{k=0}^{\infty} \sum_{l=0}^{\infty} \sum_{n, p, q=0}^{\infty} \frac{\varepsilon_{n}(2 k) ! \eta_{2} !\left|\sin ^{\eta_{1}} \alpha\right| \cos ^{2} \alpha}{(k !)^{2} l ! p ! q !(p+n) !(q+2 n) !} \\
& \frac{\gamma^{q+n} \exp (-\gamma)}{2^{\eta_{1}+\eta_{2}+1}}\left[\sin ^{2} \varphi+\cos ^{2}(\alpha+\varphi)\right]^{q+n}
\end{aligned}
$$

and the rearrangement of terms yields

$$
\begin{aligned}
|P(\epsilon \mid \varphi)| & <\cos ^{2} \alpha \exp (-\gamma) \sum_{k=0}^{\infty} \frac{\Gamma\left(k+\frac{1}{2}\right)}{\sqrt{\pi} k !} \sin ^{2 k} \alpha \\
& \times \sum_{n, p, q=0}^{\infty} \frac{\varepsilon_{n} \eta_{2} !\left|\sin ^{2 p+n} \alpha\right| \gamma^{q+n}}{p ! q !(p+n) !(q+2 n) ! 2^{4 p+3 n+q+1}} \\
& \times\left[\sin ^{2} \varphi+\cos ^{2}(\alpha+\varphi)\right]^{q+n} \\
& \times \sum_{l=0}^{\infty} \frac{(2 p+2 n+q+l) !}{l ! 2^{l}}
\end{aligned}
$$

where we have used the identity $(2 n) !=\frac{2^{2 n} n !}{\sqrt{\pi}} \Gamma\left(n+\frac{1}{2}\right), n=$ $0,1, \ldots$ A careful inspection yields the summation of first infinite series as ${ }_{1} F_{0}\left(\frac{1}{2} ;-; \sin ^{2} \alpha\right)(=\sec \alpha)$ and an application of (23) gives the last infinite summation as $(2 p+$ $2 n+q) ! 2^{2 p+2 n+q+1}$. Upon substitution of those values in (24) results in

$$
\begin{array}{r}
|P(\epsilon \mid \varphi)|<|\cos \alpha| \exp (-\gamma) \sum_{n, p, q=0}^{\infty} \frac{\varepsilon_{n}(2 p+2 n+q) !}{p ! q !(p+n) !(q+2 n) !} \\
\frac{\left|\sin ^{2 p+n} \alpha\right| \gamma^{q+n}}{2^{4 p+3 n+q+1}}\left[\sin ^{2} \varphi+\cos ^{2}(\alpha+\varphi)\right]^{q+n}
\end{array}
$$

which is the result we get if term by term integration is performed on $\left|f_{R_{1}}^{u}\left(r_{1}\right)\right|$ over the span $[0, \infty)$. One should note that $\left|f_{R_{1}}^{u}\left(r_{1}\right)\right|$ stands for the right side of (21) corresponding to our case of interest. Following (22) we can upper bound $\left|f_{R_{1}}^{u}\left(r_{1}\right)\right|$ as

$$
\begin{aligned}
\left|f_{R_{1}}^{u}\left(r_{1}\right)\right| & <|\sec \alpha| \exp (-\gamma) r_{1} \\
& \times \exp \left\{-\frac{(1-|\sin \alpha|) \sec ^{2} \alpha}{2} r_{1}^{2}\right\} \\
& \times I_{0}\left[\sec \alpha \sqrt{2 \gamma\left(\sin ^{2} \varphi+\cos ^{2}(\varphi+\alpha)\right)} r_{1}\right]
\end{aligned}
$$

and now (25) becomes

$$
|P(\epsilon \mid \varphi)|<\int_{0}^{\infty}\left|f_{R_{1}}^{u}\left(r_{1}\right)\right| d r_{1} .
$$

The term by term integration is justified here, since that series also converges uniformly. Thereafter with the help of (26) and [20, Appendix 1, eq. 2.4.13]

$$
\int_{0}^{\infty} z \exp \left(-\lambda z^{2}\right) I_{\mu}(\alpha z) d z=\frac{1}{2 \lambda} \exp \left(\frac{\alpha^{2}}{4 \lambda}\right)
$$

we can upper bound the inequality (27) as

$$
|P(\epsilon \mid \varphi)|<\frac{|\cos \alpha| \exp (-\gamma)}{(1-|\sin \alpha|)} e^{\frac{\left[\sin ^{2} \varphi+\cos ^{2}(\varphi+\alpha)\right]}{1-|\sin \alpha|} \gamma}
$$

which completes our proof.

\section{ACKNOWLEDGMENT}

The authors would like to thank the associate editor and the anonymous reviewers for their critical comments that greatly improved this paper and for bringing [4] to our attention. The first author would like to thank the government of Finland and the former AIT-Finnish project director late Prof. A. B. Sharma for the doctoral scholarship provided to him. 


\section{REFERENCES}

[1] M. Valkama, M. Renfors, and V. Koivunen, "Advanced methods for I/Q imbalance compensation in communication receivers," IEEE Trans. Signal Processing, vol. 49, no. 10, pp. 2335-2344, Oct. 2001.

[2] X. Huang, "On transmitter gain/phase imbalance compensation at receiver," IEEE Commun. Lett., vol. 4, no. 11, pp. 363-366, Nov. 2000.

[3] J. K. Cavers and M. W. Liao, "Adaptive compensation for imbalance and offset losses in direct conversion transceivers," IEEE Trans. Veh. Technol., vol. 42, no. 4, pp. 581-588, Nov. 1993.

[4] P. Beckmann, Probability in Communication Engineering. Harcourt, Brace \& World, Inc., 1967.

[5] R. Pawula, S. Rice, and J. Roberts, "Distribution of the phase angle between two vectors perturbed by gaussian noise," IEEE Trans. Commun., vol. 30, no. 8, pp. 1828-1841, Aug. 1982.

[6] M. K. Simon and D. Divsalar, "Some new twists to problems involving the gaussian probability integral," IEEE Trans. Commun., vol. 46, no. 2, pp. 200-210, Feb. 1998.

[7] R. F. Pawula, "Distribution of the phase angle between two vectors perturbed by gaussian noise II," IEEE Trans. Veh. Technol., vol. 50, no. 2, pp. 576-583, Mar. 2001.

[8] S. Park and S. H. Cho, "SEP performance of coherent MPSK over fading channels in the presence of phase/quadrature error and i-q gain mismatch," IEEE Trans. Commun., vol. 53, no. 7, pp. 1088-1091, July 2005.

[9] P. K. Vitthaladevuni and M.-S. Alouini, "Effects of imperfect phase and timing synchronization on the bit-error rate performance of PSK modulations," IEEE Trans. Commun., vol. 53, no. 7, pp. 1096-1099, July 2005.
[10] G. K. Karagiannidis, D. A. Zogas, and S. A. Kotsopoulos, "On the multivariate Nakagami- $m$ distribution with exponential correlation," IEEE Trans. Commun., vol. 51, no. 8, pp. 1240-1244, Aug. 2003.

[11] G. K. Karagiannidis, D. A. Zogas, and S. A. Kotsopoulos, "An efficient approach to multivariate Nakagami- $m$ distribution using Green's matrix approximation," IEEE Trans. Wireless Commun., vol. 2, no. 5, pp. 883889, Sep. 2003.

[12] C. W. Helstrom, "The resolution of signals in white, Gaussian noise," in Proc. IRE, Sept. 1955, pp. 1111-1118

[13] A. Viterbi, "Optimum detection and signal selection for partially coherent binary communication," IEEE Trans. Inform. Theory, vol. 11, pp. 239-246, Apr. 1965.

[14] M. Nesenbergs, "Optimum reception of coded multiple frequency keying in presence of random variable phases," IEEE Trans. Commun., vol. 19, pp. 707-711, Oct. 1971.

[15] G. N. Watson, A Treatise on the Theory of Bessel Functions. Cambridge, U.K.: Cambridge Univ. Press, 1944.

[16] S Haykin, Digital Communications. John Wiley and Sons, 1988.

[17] J. G. Proakis, Digital Communications, 3rd ed. McGraw-Hill, 1995.

[18] I. S. Gradshteyn and I. M. Ryzhik, Tables of Integrals, Series and Products, 4th ed. New York: Academic Press, 1980.

[19] L. C. Andrews, Special Functions of Mathematics for Engineers, 2nd ed. Bellignham, MA: SPIE Press, 1998.

[20] K. S. Miller, Multidimensional Gaussian Distributions. John Wiley, 1964. 